\title{
ESTIMATING PROBABILITY DENSITY FUNCTIONS AND ENTROPIES OF CHUA'S CIRCUIT USING B-SPLINE FUNCTIONS
}

\author{
F. A. SAVACI* and M. GÜNGÖR ${ }^{\dagger}$ \\ Department of Electric and Electronics Engineering, \\ Izmir Institute of Technology, Gulbahce Koyu, \\ 35430, Izmir, Turkey \\ *acarsavaci@iyte.edu.tr \\ ${ }^{\dagger}$ mesutgungor@iyte.edu.tr
}

Received December 31, 2010; Revised May 6, 2011

\begin{abstract}
In this paper, first the probability density functions (PDFs) of the states of Chua's circuit have been estimated using B-spline functions and then the state entropies of Chua's circuit with respect to the bifurcation parameter have been obtained. The results of the proposed B-spline density estimator have been compared with the results obtained from the Parzen density estimator.
\end{abstract}

Keywords: Chua's circuit; probability density functions; entropy; B-spline density estimator; Parzen density estimator; generalized Chua's circuit.

\section{Introduction}

The nonparametric density estimation finds wide interest in many signal processing applications [Scott, 1992; Hwang et al., 2002; Kehtarnavaz \& Nakamura, 1998] and also in control applications [Wang, 2000]. In the control of stochastic dynamical systems, to reshape the PDFs of the output is necessary for tracking the target PDFs [Sun, 2006; Guo et al., 2008; Hong et al., 2005; Elbeyli et al., 2005], therefore online estimation of the output PDF is important and in [Guo \& Wang, 2005], B-spline approximation of the output PDFs is used to design PID controller for controlling the stochastic dynamics. The PDF estimation can also be used to test the efficiency of the designed chaotic circuits with prescribed random properties given in [Chua et al., 1990]. PDF estimation has also been studied for blind source separation in [Nakayama et al., 2003] and PDF matching problem has been studied for blind equalization in [Lázaro et al., 2005].

The state density estimation of dynamical systems is of importance not only in the stochastic dynamical systems but also in the deterministic dynamical systems exhibiting chaotic dynamics when the uncertainties in the initial conditions are considered as the initial densities and hence chaotic dynamics can be investigated through the evolutions of the PDFs governed by Fokker-PlanckKolmogorov (FPK) equation [Lasota \& Mackey, 1994; Günel, 2004; Günel \& Savaci, 2005a, 2006]. The compactly supported polynomials (CSPs) defined over the ellipsoidal regions have been used to estimate the PDFs of the chaotic attractors since the attractors of chaotic dynamics lie in the compact subspace of the state space [Günel \& Savaci, 2006] and the efficient results are comparable to the methods which used the mixture of Gaussian distributions [Iyengar \& Dash, 1978; Er, 1998] or exponential distributions [Er, 1999, 2000].

In this paper, for estimating the state densities of Chua's circuit [Chua et al., 1986] and of Generalized Chua Circuit (GCC) [Suykens et al., 1997], inspired from the idea of using CSPs [Günel \& Savaci, 2006], we have used B-splines since they 
also have compact supports defined by nondecreasing knot sequences obtained simply from the simulations or observed data. Contrary to the Gaussian mixture model given in [Kehtarnavaz \& Nakamura, 1998], these estimated PDFs have been compared with the estimated PDFs of Chua's circuit obtained by Parzen density estimator as in [Günel, 2004].

Our second aim in this paper after estimating the PDFs of Chua's circuit by using B-splines is to evaluate the entropies of Chua's circuit with respect to the bifurcation parameter. The entropy evaluation of chaotic attractors is of interest especially in cryptography applications. As a promising application of $n$-scroll attractors, Yalcin et al. [2004] have introduced true random number generator (TRNG) based on a double scroll attractor and in [Yalcin, 2007], it was shown that by increasing the number of scrolls, the entropy of TRNG could be increased. The entropies of Chua's circuit have been estimated by using Parzen density estimator in [Günel \& Savaci, 2005b; Günel, 2004] while in our study the evaluations of the entropies of Chua's circuit are based on the estimated PDFs obtained from the data of simulations by using B-spline estimator. The entropy of Chua's circuit has also been studied and a formula for the topological entropy has been given in [Yang \& Li, 2005].

In the organization of this paper, first the B-spline functions and its properties will be briefly introduced in Sec. 2.1, then our proposed density estimation method using B-splines is explained in Sec. 2.2. In Sec. 3.1, the state densities of Chua's circuit are estimated based on this proposed method and the results are compared with the estimated PDFs obtained by Parzen's density estimator and in Sec. 3.2, the entropies of Chua's circuit are obtained with respect to bifurcation parameter $\alpha$ using PDFs obtained by B-spline density estimator and Parzen density estimator.

\section{B-Splines and Density Estimation}

The spline functions defined in [Curry \& Schoenberg, 1966] are used to approximate the PDFs by a linear combination of B-splines [Zong \& Lam, 1998; Guo \& Wang, 2005; Zong, 2006].

\subsection{B-splines and its properties}

Let $x_{i} \in R, i=0,1, \ldots, N$ be a nondecreasing knot sequence. Then one can construct $k$ th order B-spline $B_{i}^{k}(x)$ from the recursion formula [De Boor, 1972] as

$$
\begin{aligned}
B_{i}^{k}(x)= & \frac{x-x_{i}}{x_{i+k-1}-x_{i}} B_{i}^{k-1}(x) \\
& +\frac{x_{i+k}-x}{x_{i+k}-x_{i+1}} B_{i+1}^{k-1}(x) \quad k=2,3, \ldots
\end{aligned}
$$

and the first order B-spline is the indicator function $I(\cdot)$ defined as

$$
B_{i}^{1}(x)=I_{\left[x_{i}, x_{i+1}\right)}(x) \doteq \begin{cases}1 & x \in\left[x_{i}, x_{i+1}\right) \\ 0 & \text { otherwise }\end{cases}
$$

The recursive relation in Eq. (1) can also be expressed as the repeated $k$ convolution of B-spline of order one [Unser et al., 1993],

$$
B_{i}^{k}(x)=\underbrace{B_{i}^{1} * B_{i}^{1} * \cdots * B_{i}^{1}}_{k \text { times }}
$$

where $*$ denotes the convolution operation. From Eq. (1), the widely used third order B-spline can be explicitly written as

$$
\begin{aligned}
B_{i}^{3}(x)= & \frac{\left(x-x_{i}\right)^{2}}{\left(x_{i+1}-x_{i}\right)\left(x_{i+2}-x_{i}\right)} I_{\left[x_{i}, x_{i+1}\right)}(x) \\
& +\frac{1}{x_{i+2}-x_{i+1}}\left[\frac{\left(x-x_{i}\right)\left(x_{i+2}-x\right)}{\left(x_{i+2}-x_{i}\right)}\right. \\
& \left.+\frac{\left(x-x_{i+1}\right)\left(x_{i+3}-x\right)}{\left(x_{i+3}-x_{i+1}\right)}\right] I_{\left[x_{i+1}, x_{i+2}\right)}(x) \\
& +\frac{\left(x_{i+3}-x\right)^{2}}{\left(x_{i+3}-x_{i+1}\right)\left(x_{i+3}-x_{i+2}\right)} \\
& \times I_{\left[x_{i+2}, x_{i+3}\right)}(x) .
\end{aligned}
$$

Because of its following properties B-splines can be efficiently used to estimate the PDFs:

(i) The sequence of $\left\{B_{i}^{k}\right\}_{i=1}^{n}$ of B-splines of order $k$ for the nondecreasing knot sequence $\Delta=$ $\left\{x_{1}, \ldots, x_{n+k}\right\}$ constitutes a basis for the space of piecewise polynomials of degree $\leq k$.

(ii) Non-negative $B_{i}^{k}(x) \mathrm{s}$ have compact support (i.e. nonzero polynomial on $x_{i} \leq x \leq x_{i+k}$, otherwise it is identically equal to zero).

(iii) $B_{i}^{k}$ s are normalized i.e.

$$
\sum_{i=1}^{N} B_{i}^{k}(x) \equiv 1, \quad x \in\left[x_{k}, x_{N+1}\right] .
$$




\subsection{One-dimensional (1D) density estimation using B-splines}

In the following sequel we have developed a simple method based on the expectations of B-spline functions and the inner products of the B-spline functions whereas the estimation of the PDF in [Zong \& Lam, 1998; Zong, 2006] has been done based on the maximum likelihood method.

One-dimensional true probability density function $f(x)$ is approximated as $\hat{f}(x)$ using $N$ number of third order B-splines

$$
f(x) \cong \hat{f}(x)=\sum_{i=1}^{N} a_{i} B_{i}^{3}(x)
$$

where the unknown coefficients $a_{i}$ 's are the contributions of each B-spline to the approximated probability density function.

For the density estimation of streaming data of a nonstationary process in [Caudle \& Wegman, 2009], the simple idea introduced by Cencov [1962] was used to find the coefficients of the orthogonal series from expectations of basis functions. We have also used this simple idea to determine the unknown coefficients $a_{i}$ s of the estimated PDF in terms of the nonorthogonal B-splines as: multiplying both sides of Eq. (5) with $B_{j}^{3}(x)$ and integrating over the interval $\left[x_{\min }, x_{\max }\right]$ obtained from the attractor, one has,

$$
\int_{x_{\min }}^{x_{\max }} B_{j}^{3}(x) f(x) d x=\sum_{i=1}^{N} a_{i} k_{i j}, \quad j=1, \ldots, N
$$

where the inner products " $k_{i j}$ " of B-splines can be computed explicitly as

$$
k_{i j}=\int_{x_{i}}^{x_{i}+m h} B_{i}^{3}(x) B_{j}^{3}(x) d x
$$

with the spacing $h \doteq x_{i+1}-x_{i}$ and $m=3$ for the third order B-splines.

The left-hand side of Eq. (7) is the expectation of $B_{j}^{3}(x)$, " $E\left\{B_{j}^{3}(x)\right\}$ " which can be approximated as

$$
E\left\{B_{j}^{3}(x)\right\} \cong \bar{b}_{j} \doteq \frac{1}{n} \sum_{l=1}^{n} B_{j}^{3}\left(x_{l}\right)
$$

where $x_{l}$ s are the observed data and $n$ is the number of data. Then the unknown coefficients $a_{i}$ s can be calculated from the following linear algebraic equation obtained from Eq. (6),

$$
\sum_{i=1}^{N} a_{i} k_{i j}=\bar{b}_{j}, \quad j=1, \ldots, N
$$

where the nonsingular coefficient band matrix $K \doteq$ $\left[k_{i j}\right]$ is defined as,

$$
k_{i j} \doteq \begin{cases}h I_{0}, & |i-j|=0 \\ h I_{1}, & |i-j|=1 \\ h I_{2}, & |i-j|=2 \\ 0, & |i-j|>3 .\end{cases}
$$

The values of $I_{0}, I_{1}, I_{2}$ can be found in the table of [Schumaker, 2007, p. 137] which are determined as

$$
\begin{aligned}
j=0, \quad k_{i 0} & =\int_{x_{i}}^{x_{i}+3 h} B_{i}^{3}(x) B_{i}^{3}(x) d x \\
& =h I_{0}^{3,3}=h \frac{66}{120} \\
j=1, \quad k_{i 1} & =\int_{x_{i}}^{x_{i}+3 h} B_{i}^{3}(x) B_{i+1}^{3}(x) d x \\
& =h I_{1}^{3,3}=h \frac{26}{120} \\
j=2, \quad k_{i 2} & =\int_{x_{i}}^{x_{i}+3 h} B_{i}^{3}(x) B_{i+2}^{3}(x) d x \\
& =h I_{2}^{3,3}=h \frac{1}{120} .
\end{aligned}
$$

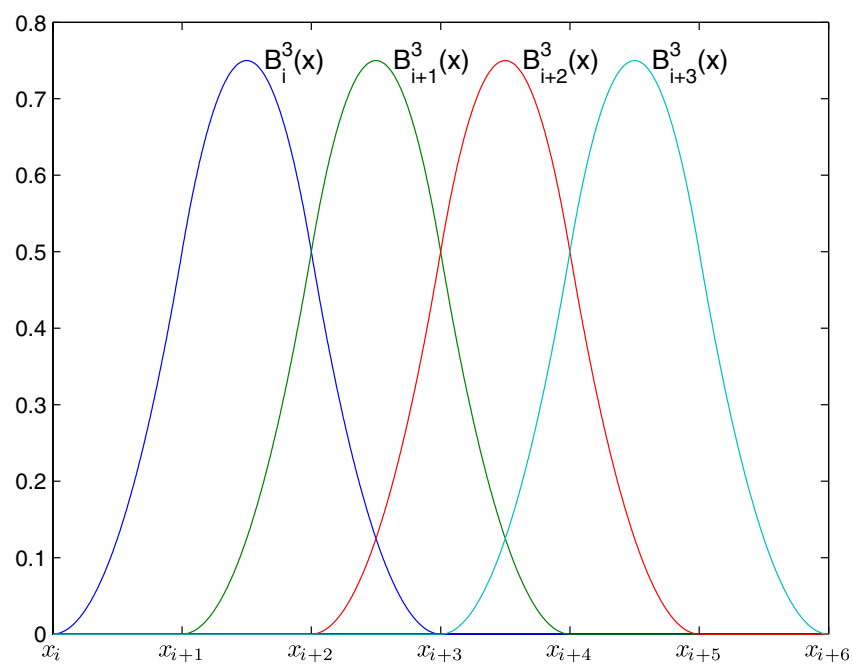

Fig. 1. The inner product of $B_{i}^{3}(x)$ and $B_{i+3}^{3}(x)$ is zero. 
Note that $k_{i j}=0 j>2$ because there is no intersection of the $i$ th and $((i+3)$ th, $(i+4)$ th, $(i+5)$ th $, \ldots,(i+(N$-order $))$ th $)$ B-splines which can be seen in Fig. 1. Then one can determine the unknown coefficients vector a using Eq. (9) as,

$$
\mathbf{a}=\left(K^{t}\right)^{-1} \mathbf{b}
$$

where

$$
\mathbf{a} \doteq\left[a_{1}, a_{2}, \ldots, a_{N}\right]^{t}
$$

and $\mathbf{b}$ is the vector of estimated means $\bar{b}_{j}$

$$
\mathbf{b}^{t} \doteq\left[\bar{b}_{1}, \bar{b}_{2}, \ldots, \bar{b}_{N}\right] \text {. }
$$

In order that the estimated $\operatorname{PDF}$ " $\hat{f}(x)$ " be a proper probability density function, the integral of the estimated density over the observation domain (i.e. the interval $\left.\left[x_{\min }, x_{\max }\right]\right)$ should be equal to unity which yields the following constraint:

$$
\sum_{i=1}^{N} a_{i} c_{i}=1
$$

where

$$
c_{i} \doteq \int_{x_{\min }}^{x_{\max }} B_{i}^{3}(x) d x=\frac{x_{i}-x_{i-3}}{3}=h
$$

as given in [Zong, 2006]. The constraint given in Eq. (14) implicitly depends on the number of B-splines.

\subsection{Two-dimensional (2D) density estimation using B-splines}

Two-dimensional (2D) estimation can be found by using 2D B-splines as given in [Zong, 2006]

$$
f(x, y) \cong \hat{f}(x, y)=\sum_{i=1}^{M} \sum_{j=1}^{N} a_{i j} B_{i}^{3}(x) B_{j}^{3}(y)
$$

by multiplying both sides with $B_{k}^{3}(x) B_{l}^{3}(y)$, and integrating

$$
\begin{gathered}
\int_{x_{\min }}^{x_{\max }} \int_{y_{\min }}^{y_{\max }} \hat{f}(x, y) B_{k}^{3}(x) B_{l}^{3}(y) d y d x \\
=\sum_{i=1}^{M} \sum_{j=1}^{N} a_{i j} t_{i k} z_{j l}
\end{gathered}
$$

the left-hand side is the expected value of the product $B_{k}^{3}(x) B_{l}^{3}(y)$ i.e.

$$
\begin{aligned}
E\left\{B_{k}^{3}(x) B_{l}^{3}(y)\right\} & \\
& \doteq \bar{b}_{k l} \cong \frac{1}{n^{2}} \sum_{r=1}^{n} \sum_{u=1}^{n} B_{k}^{3}\left(x_{u}\right) B_{l}^{3}\left(y_{r}\right)
\end{aligned}
$$

where the inner product terms appearing on the right-hand side of Eq. (17) are defined as,

$$
t_{i k} \doteq \int_{x_{i}}^{x_{i}+m h_{x}} B_{i}^{3}(x) B_{k}^{3}(x) d x
$$

and

$$
z_{j l} \doteq \int_{y_{j}}^{y_{j}+m h_{y}} B_{j}^{3}(y) B_{l}^{3}(y) d y
$$

where the increments are defined as $h_{x} \doteq x_{i+1}-x_{i}$ and $h_{y} \doteq y_{j+1}-y_{j}$ and the values of $t_{i k}$ and $z_{j l}$ are determined using Eq. (10).

Then one can find the coefficient matrix $A$ using Eqs. (17) and (18) as,

$$
A=\left(T^{t}\right)^{-1} B^{t} Z^{-1}
$$

where $B$ is the matrix of estimated means $\bar{b}_{k l}$, $k=1, \ldots, N$ and $l=1, \ldots, M$. In order that the estimated joint probability density " $\hat{f}(x, y)$ " be a proper probability density function, the integral of the estimated density over the observation domain (i.e. the interval $x \in\left[x_{\min }, x_{\max }\right]$ and $y \in$ $\left.\left[y_{\min }, y_{\max }\right]\right)$ should be equal to unity which yields the constraint:

$$
\sum_{i=1}^{M} \sum_{j=1}^{N} a_{i j} c_{i j}=1
$$

where

$$
\begin{aligned}
c_{i j} & \doteq \int_{x_{\min }}^{x_{\max }} B_{i}^{3}(x) d x \int_{y_{\min }}^{y_{\max }} B_{j}^{3}(y) d y \\
& =\frac{\left(x_{i}-x_{i-3}\right)}{3} \frac{\left(y_{j}-y_{j-3}\right)}{3}=h_{x} h_{y} .
\end{aligned}
$$

\subsection{Marginal densities}

From the estimated 2D density $f(x, y)$ one can also find the marginal densities $\hat{f}_{x}(x)$ and $\hat{f}_{y}(y)$ as below

$$
\begin{aligned}
\hat{f}_{x}(x) & =\int_{-\infty}^{\infty} \hat{f}(x, y) d y \\
& =\int_{-\infty}^{\infty} \sum_{i=1}^{M} \sum_{j=1}^{N} a_{i j} B_{i}^{3}(x) B_{j}^{3}(y) d y \\
& =\sum_{i=1}^{M} \sum_{j=1}^{N} a_{i j} B_{i}^{3}(x) \int_{-\infty}^{\infty} B_{j}^{3}(y) d y \\
& =h_{y} B_{x}^{t} \mathbf{a}
\end{aligned}
$$


where

$$
\begin{aligned}
B_{x}^{t} & \doteq\left[\begin{array}{llll}
B_{1}^{3}(x) & B_{1}^{3}(x) & \cdots & B_{N}^{3}(x)
\end{array}\right] \\
\mathbf{a} & \doteq \sum_{i=1}^{N} \mathbf{a}_{i} \\
a_{i} & \doteq\left[\begin{array}{llll}
a_{i 1} & a_{i 2} & \cdots & a_{i N}
\end{array}\right]^{t} .
\end{aligned}
$$

By following the same steps one can easily find $f_{y}(y)$ as

$$
f_{y}(y)=h_{x} B_{y}^{t} \mathbf{a} .
$$

In Sec. 3, 2D and 1D density estimations of Generalized Chua's Circuit (GCC) have been obtained. Then directly estimated 1D pdfs obtained in Sec. 2.2 have also been verified by the marginal densities in Eqs. (24) and (26) obtained from the directly estimated 2D pdfs given in Sec. 2.3.

\subsection{Parzen density estimation}

Parzen density estimation method is given in [Shanmugan \& Breipohl, 1988] as,

$$
\hat{f}(x)=\frac{1}{n h(n)} \sum_{l=1}^{n} K\left(\frac{x-x_{l}}{h}\right)
$$

where the weighting function $K(\cdot)$ and a smoothing factor $h(n)$ are defined as

$$
K\left(\frac{x-x_{l}}{h(n)}\right)=\frac{1}{\sqrt{2 \pi}} e^{-\frac{\left(x-x_{l}\right)^{2}}{2 h^{2}}}
$$

where

$$
h(n)=\frac{1}{\sqrt{n}} .
$$

In the following sequel, Parzen density estimator given in Eq. (27) have been used to compare the PDFs obtained by the proposed B-spline estimation method.

\section{PDF and Entropy Estimation of Generalized Chua's Circuit ( $\mathrm{GCC}$ )}

\subsection{PDF estimation of $G C C$}

The dynamics of GCC [Suykens et al., 1997] are given as

$$
\begin{aligned}
& \dot{x}=\alpha[y-g(x)] \\
& \dot{y}=x-y+z \\
& \dot{z}=-\beta y
\end{aligned}
$$

where

$$
\begin{aligned}
g(x)= & m_{2 q-1} x+\frac{1}{2} \sum_{i=1}^{2 q-1}\left(m_{i-1}-m_{i}\right) \\
& \times\left(\left|x+c_{i}\right|-\left|x-c_{i}\right|\right)
\end{aligned}
$$

and the number of scrolls in GCC is controlled by the parameters $q$ and $m_{i}$.

For the double scroll case the parameters are chosen as $q=c=1$ and $m_{0}=-\frac{1}{7}, m_{0}=\frac{2}{7}$ [Chua et al., 1986].

$$
\begin{aligned}
& \dot{x}=\alpha[y-g(x)] \\
& \dot{y}=x-y+z \\
& \dot{z}=-\beta y
\end{aligned}
$$

where

$$
g(x)=m_{1} x+\frac{1}{2}\left(m_{0}-m_{1}\right)(|x+1|-|x-1|) .
$$

The observation data have been obtained by numerically solving Eq. (30) with fifth order DormandPrice algorithm and fixed time steps of $\delta t=0.016$ and $t_{\max }=1100$. First 100 data points have been considered as transients and have been ignored as done in [Günel, 2004]. The relationship between the number of B-splines used and the integral of the estimated density over the observation domain is shown in Fig. 2. To estimate the PDFs properly by B-spline methods the number of B-splines " $N=30$ " have been chosen to satisfy the constraint given in Eq. (14).

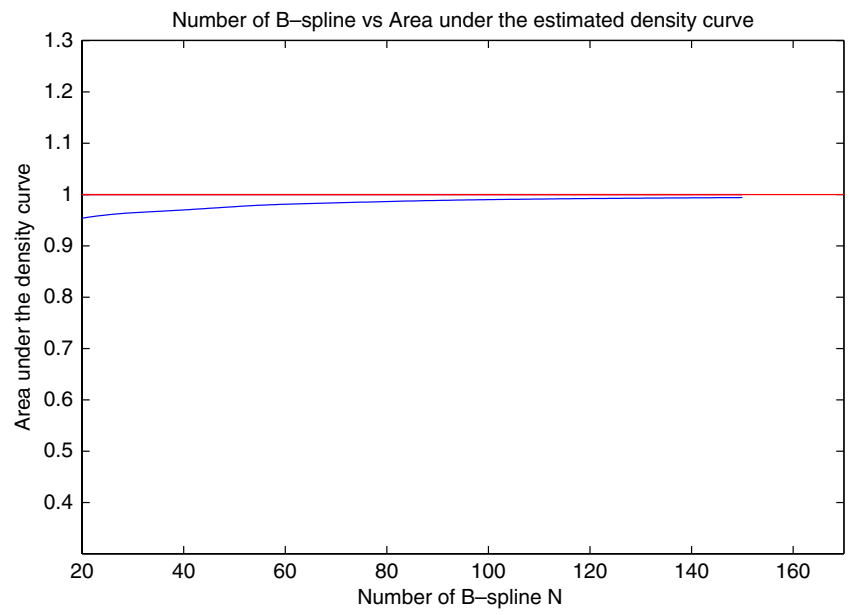

Fig. 2. Number of B-splines versus Area under the estimated PDF curve. Blue curve shows the integral under the estimated PDF. 


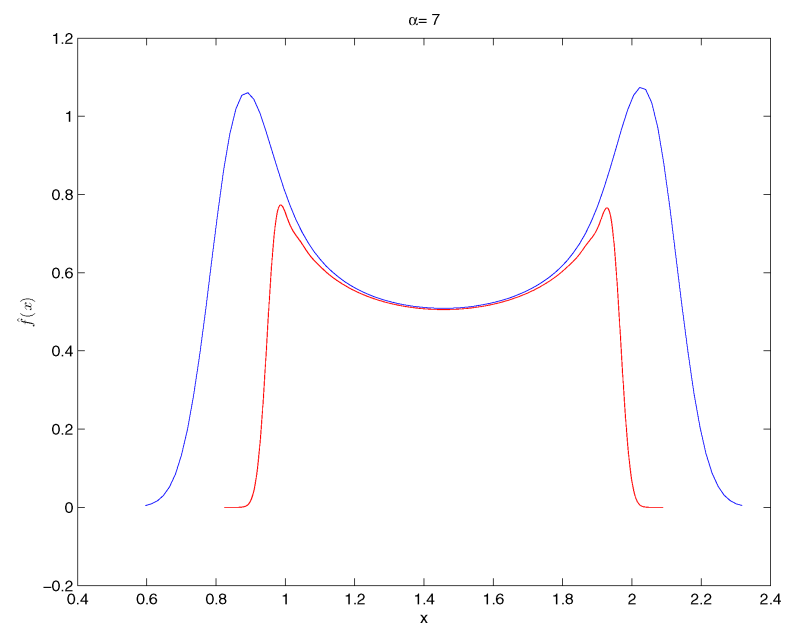

(a)

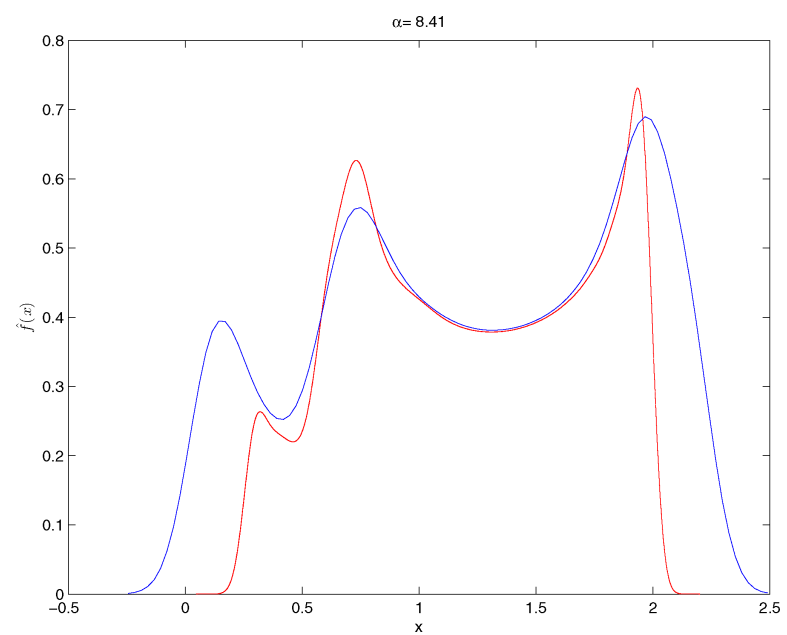

(c)

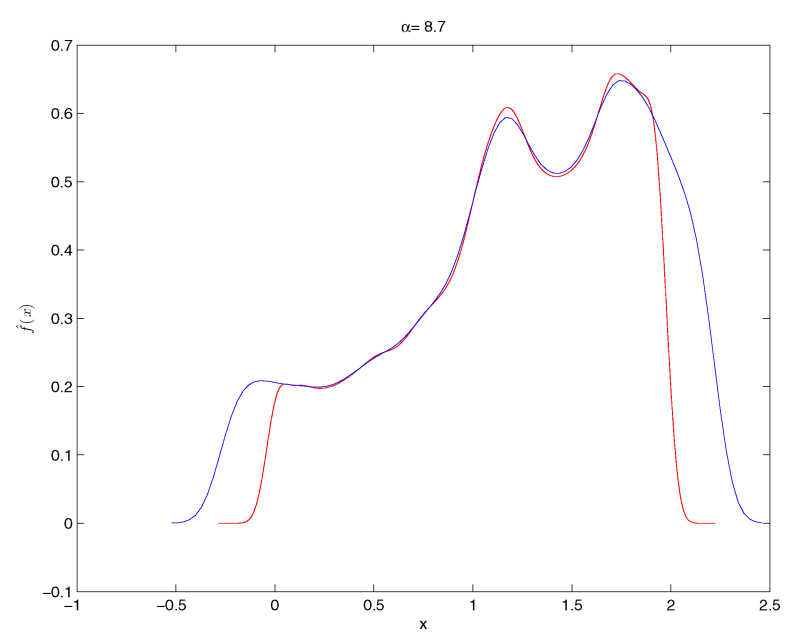

(e)

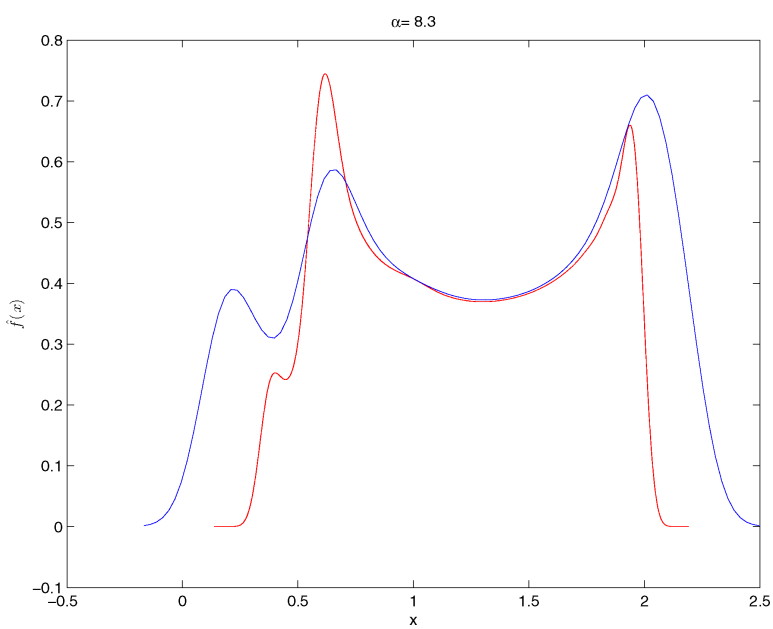

(b)

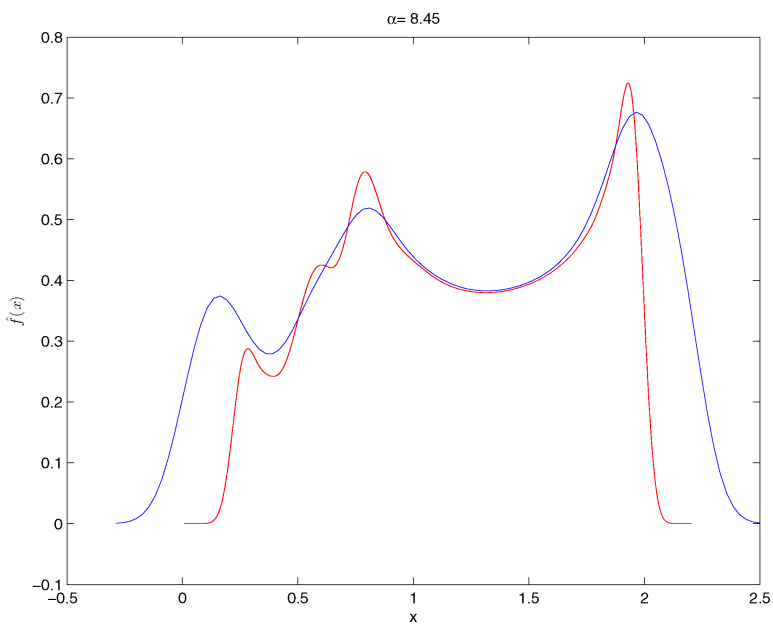

(d)

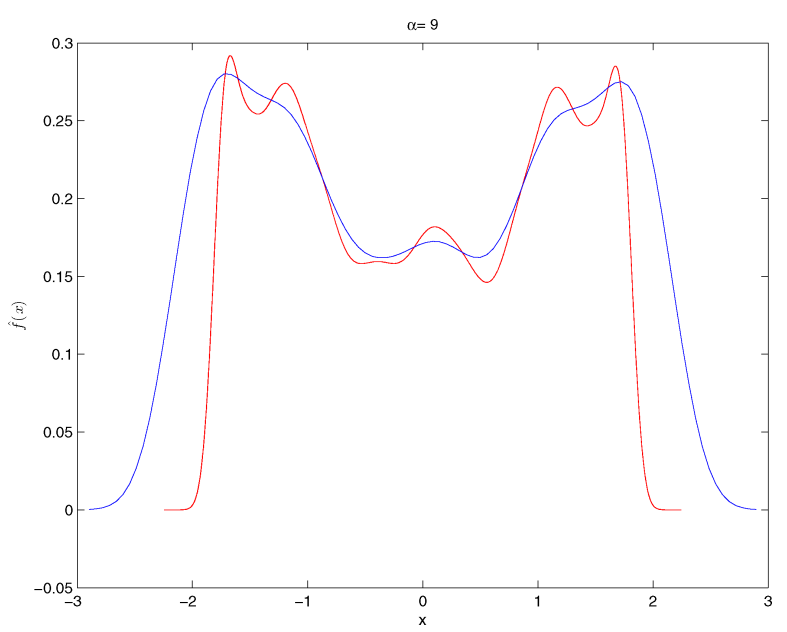

(f)

Fig. 3. Estimated densities of the state $x$ of Chua's circuit using the proposed B-spline method using 30 B-splines (red-line) and Parzen density estimation method with bandwidth obtained from the optimum bandwidth formula (blue-line). (a) $\alpha=7$ limit cycle, (b) $\alpha=8.3$ period-2, (c) $\alpha=8.41$ period- 4 , (d) $\alpha=8.45$ period-8, (e) $\alpha=8.7$ scrolls and (f) $\alpha=9$ double scroll. 
PDFs of $x$ for period-1, period-2, period-4, period- 8 scrolls and double scroll cases of Chua's circuit have been estimated by B-spline method given in Sec. 2.2 for the corresponding values of bifurcation parameter $\alpha$ and they have been shown in Fig. 3. The PDFs obtained by B-spline method and the PDFs obtained by Parzen's estimator given in Sec. 2.5 have been plotted in Fig. 3 for comparison.

In Fig. 4, PDFs obtained from both Parzen's estimator and the proposed B-spline density estimator for 3-scroll and 5-scroll dynamics of GCC have also been given. The number of scrolls can be clearly seen from the bumps of the estimated PDFs of GCC.

Although the attractors of Chua's circuit lie in the compact subset of the state space, the tails of PDFs obtained by Parzen's estimator introduce nonexisting states lying outside the attractor. This spurios states arise due to the nature of Gaussian weighting functions used in Parzen's estimator.

Since Parzen's estimator represents every observed data with a Gaussian function centered at the observed data with a standard deviation $h(n)$, it brings very intensive operation from a computational point of view compared to the B-spline estimation.

In Fig. 5, the joint probability densities belonging to the $x$ - and $z$-components of double scroll Chua's circuit have been plotted. Also 2D PDFs

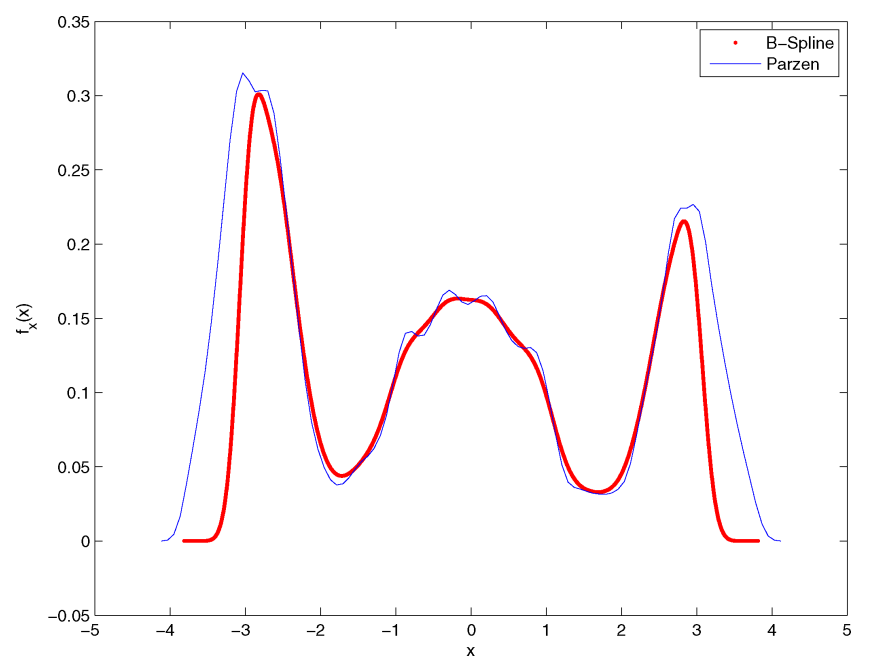

(a) for three and five scroll of GCC have been plotted in Fig. 6.

Furthermore the marginal densities obtained from the estimated 2D $\operatorname{PDF} \hat{f}(x, z)$ have been compared with the densities obtained directly from 1D PDF $\hat{f}(x)$ and $\hat{f}(z)$ in Fig. 7 .

\subsection{Entropy estimation of Chua's circuit}

In this paper, the entropies of Chua's circuit with respect to the bifurcation parameter " $\alpha$ " have been calculated using the estimated PDFs $\hat{f}(x)$ obtained in the previous section from the simulated and/or observed data using the formula given in [Fraser, 1989]

$$
\hat{h}=\lim _{n \rightarrow \infty} \frac{\hat{H}}{n}
$$

where

$$
\hat{H}=-\sum_{l=1}^{n} \hat{f}\left(x_{l}\right) \ln \left(\hat{f}\left(x_{l}\right)\right)
$$

where $n$ is the total number of data which are obtained from the computer simulations and $\hat{f}\left(x_{l}\right)$ is the individual probability of the datum $x_{l}$. For the entropy calculations, the simulations have been conducted for $\alpha$ values which have been incremented in 0.001 steps in the interval $[7.5$ 10]. If the attractor of the circuit is a fixed point then the PDF

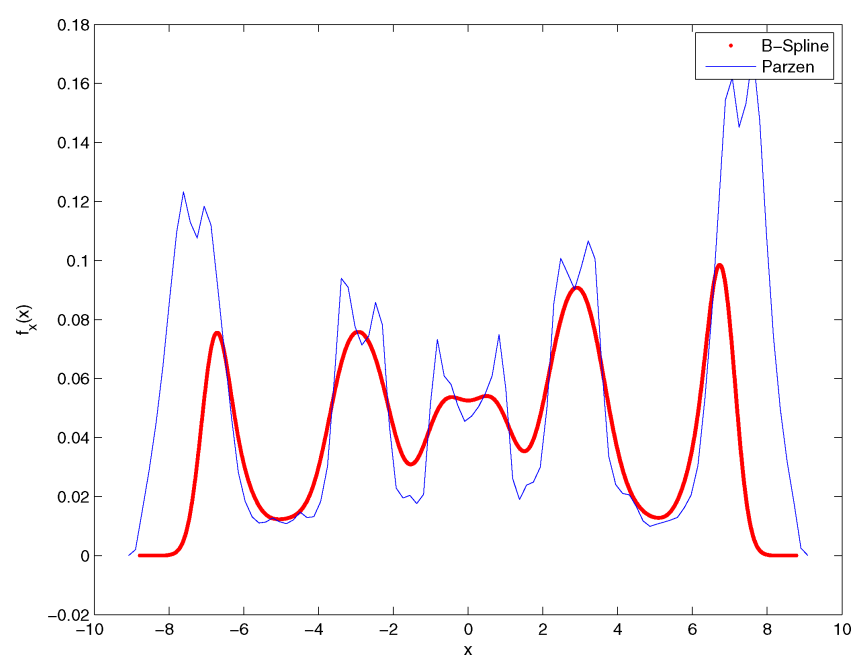

(b)

Fig. 4. 1D estimated densities of the state $x$ of Generalized Chua's Circuit using the proposed B-spline method and Parzen density estimation method. (a) 3-scroll and (b) 5-scroll. 


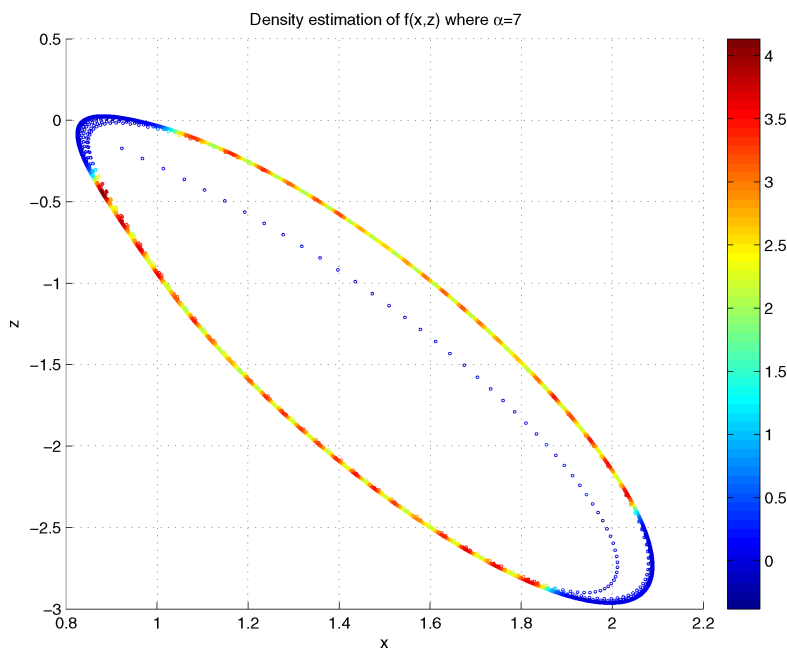

(a)

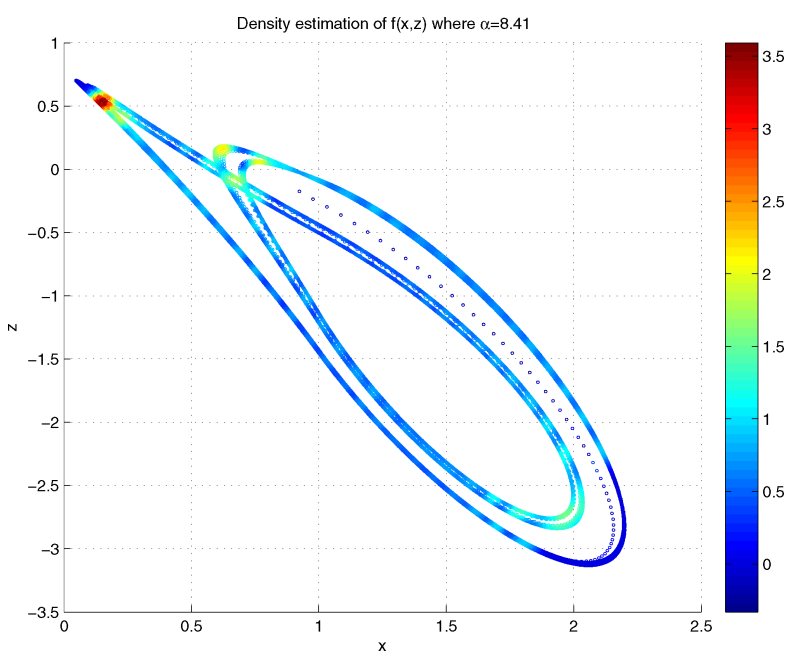

(c)

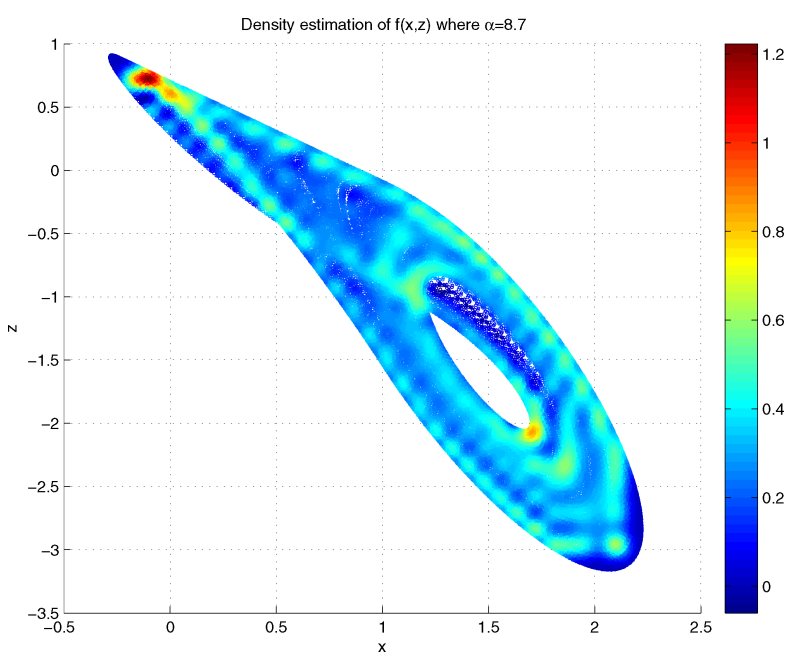

(e)

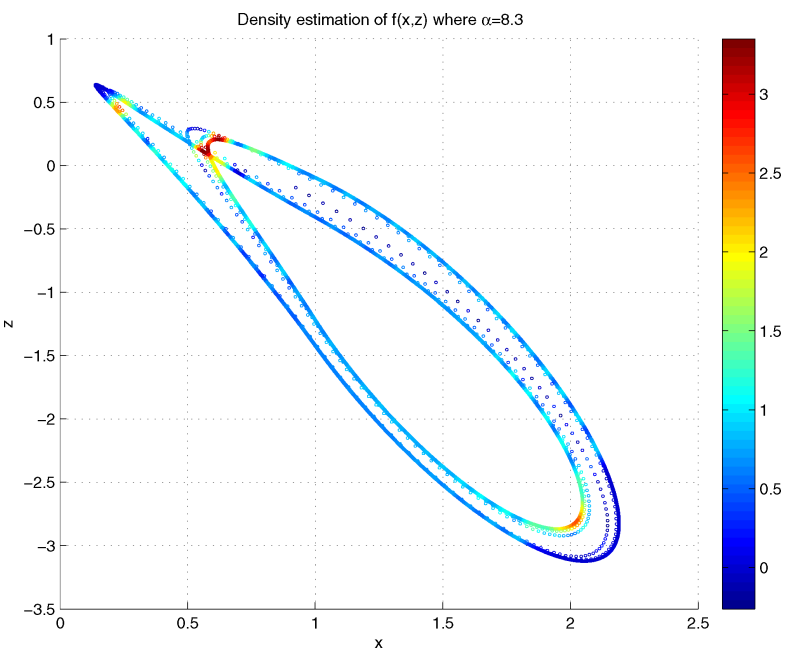

(b)

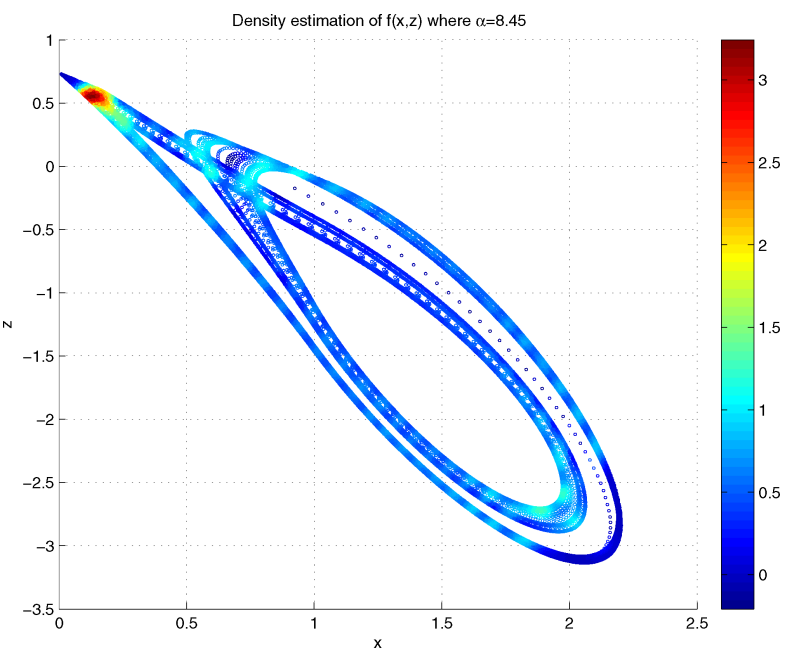

(d)

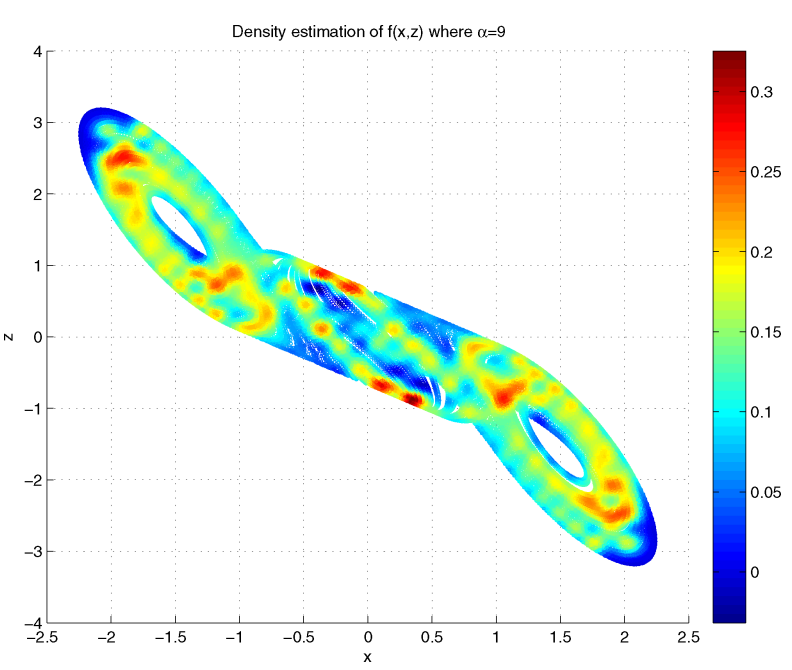

(f)

Fig. 5. Estimated densities of the states $x$ and $z$ of Chua's circuit using the proposed B-spline method: (a) $\alpha=7$ limit cycle, (b) $\alpha=8.3$ period-2, (c) $\alpha=8.41$ period- 4 , (d) $\alpha=8.45$ period- 8 , (e) $\alpha=8.7$ scrolls and (f) $\alpha=9$ double scroll. 


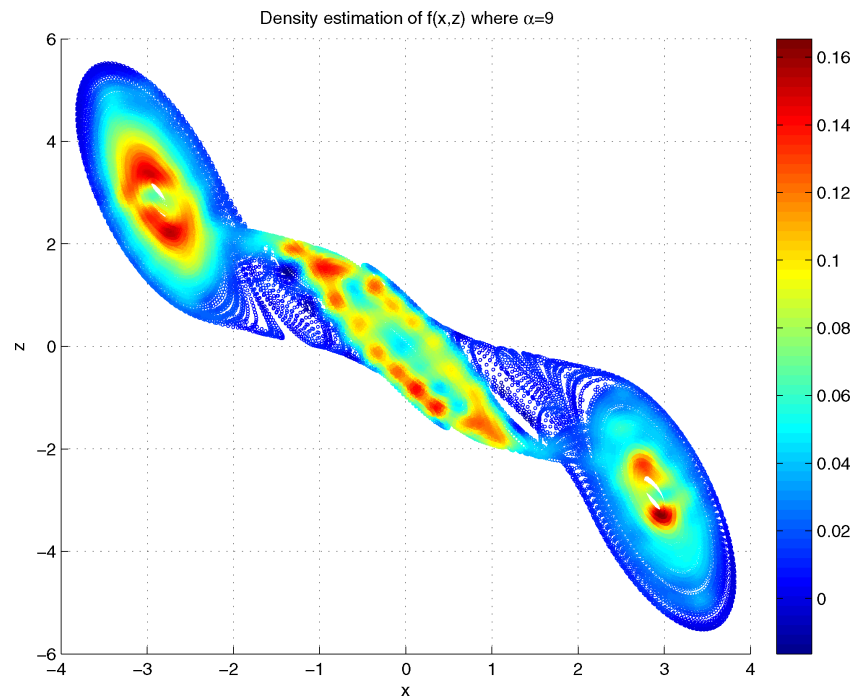

(a)

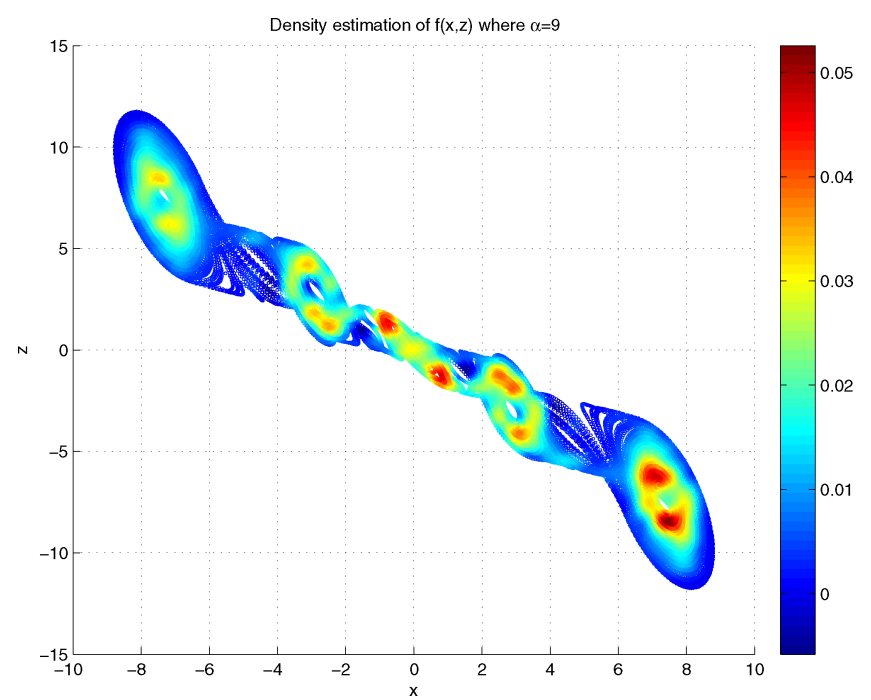

(b)

Fig. 6. 2D estimated densities of the states $x$ and $z$ of Generalized Chua's Circuit using the proposed B-spline method. The colorbar represents the values of the estimated pdf. (a) 3-scroll and (b) 5-scroll.

will consist of only one peak (i.e. unit impulse) and therefore the entropy of the fixed point will be equal to zero. Since fixed point is a trivial case it has not been shown in Fig. 8. As seen from Fig. 8 in the period-doubling route to chaos, the number of peaks of the related PDFs increases which indicates the increase in the complexity of the related attractors; hence the estimated entropies of Chua's circuit increase (which are always positive from the definition given in Eq. (35) in which $\left.0 \leq \hat{f}\left(x_{l}\right) \leq 1\right)$. Also there are sharp drops in the entropy plot at which the attractor does not exhibit complex structures in the state space.

Entropies of Chua's circuit have also been calculated by using Parzen density estimator in Fig. 9, which takes more computation time compared to the entropy calculation time using B-spline density estimator. As seen from Figs. 8 and 9, the estimated values of the entropies for B-spline and Parzen estimators are different but the similar shapes of the entropy plots prove similar bifurcation behavior.

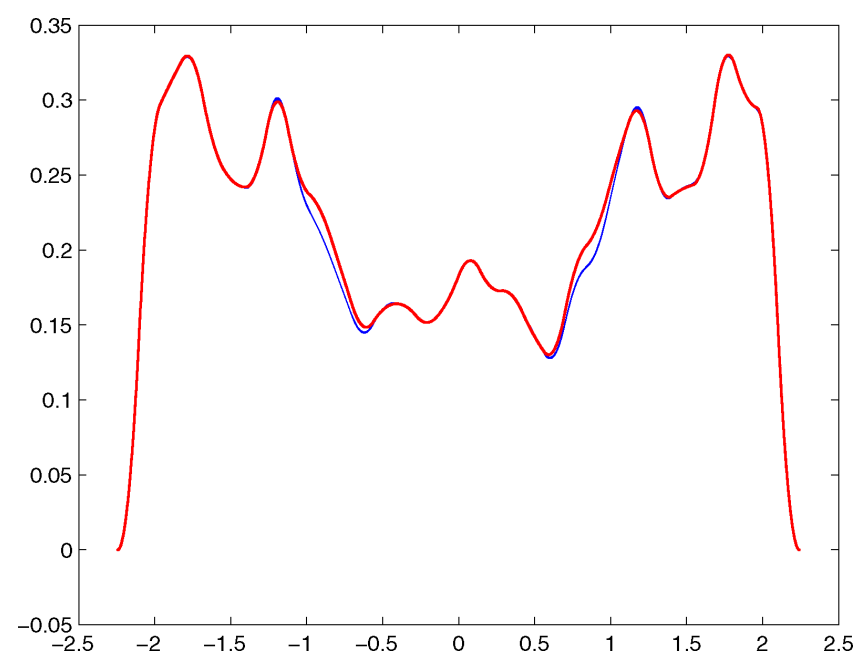

(a)

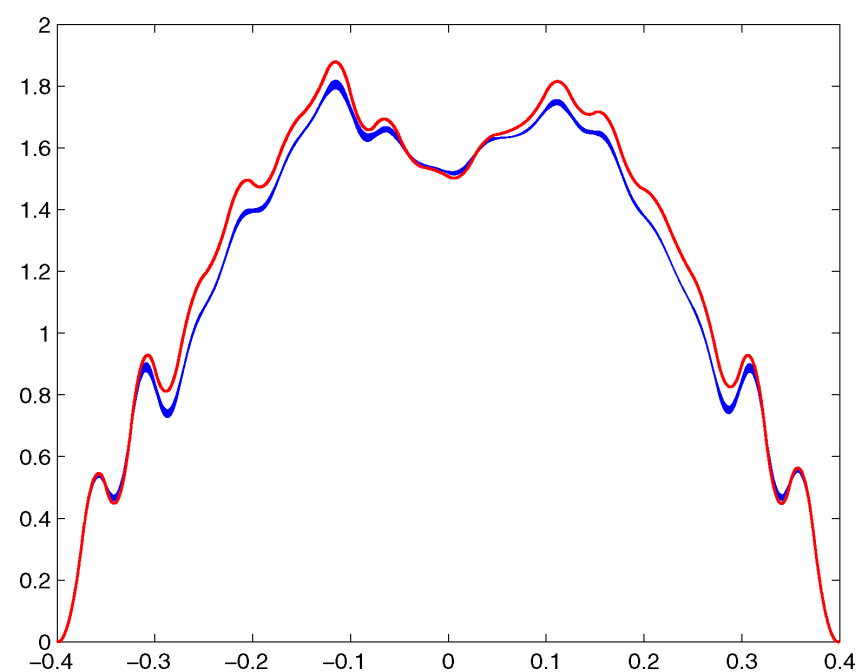

(b)

Fig. 7. Marginal densities compared with the 1D B-spline density estimation of (a) $f_{x}(x)$ and (b) $f_{y}(y)$ red curve shows $1 \mathrm{D}$ pdf estimation and the blue one is the marginal estimation calculated from the joint pdf $\hat{f}(x, y)$. 


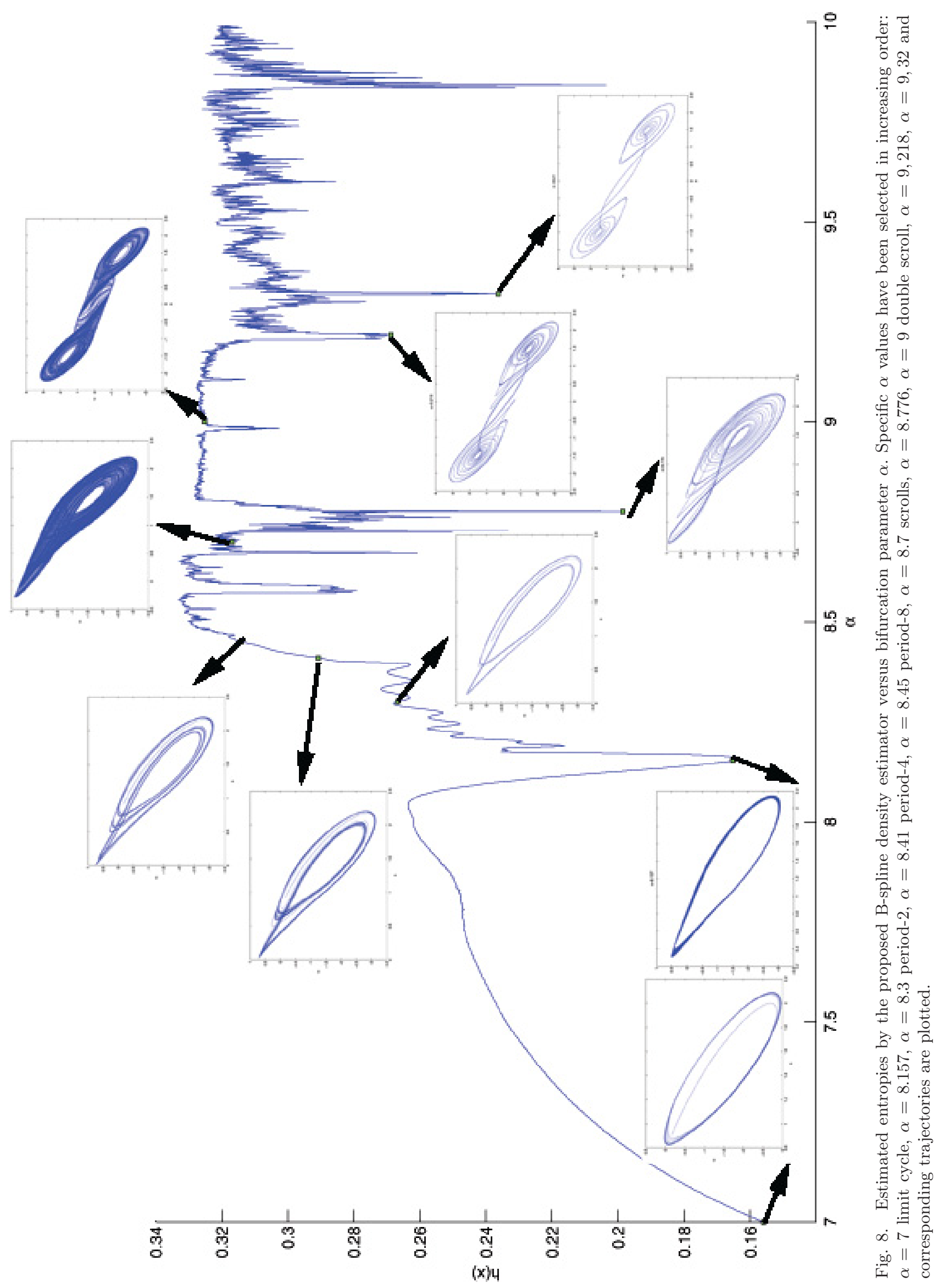




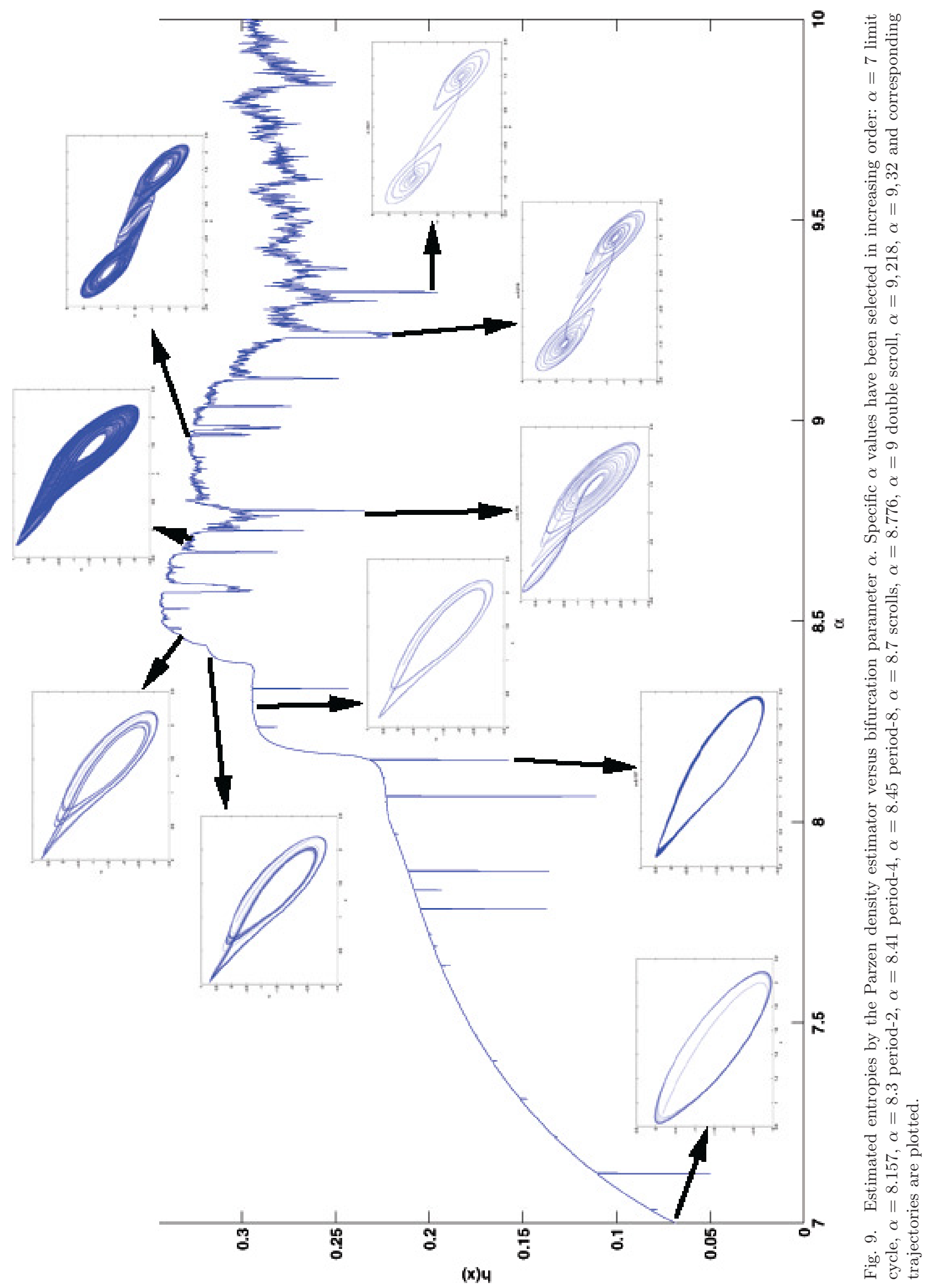

1250107-11 
We have also computed the approximate entropy (ApEn) defined in [Pincus, 1991] which is obtained by fixing some parameters of the correlation dimension given in the Eckmann-Ruelle (E-R) entropy formula [Eckmann \& Ruelle, 1985]. Although it is claimed in [Pincus, 1991] that ApEn distinguishes the complexity of the underlying dynamics compared to the E-R entropy, in our calculations ApEn did not show similar entropy behaviors obtained by B-spline estimator and Parzen estimator.

Our PDF based entropy evaluation does not also need the estimation of Lyapunov exponents to calculate the Kolmogorov-Sinai (K-S) entropy given in [Pesin, 1977].

\section{Conclusion}

The proposed B-spline Density Estimation method has been applied to estimate the state PDFs of Chua's circuit. Since the B-splines are simple polynomials defined on compact sets, this method in computational aspects has advantages compared to the Parzen's estimator which uses Gaussian distributions with long tails bringing in the spurios states which do not exist in attractors. Therefore for the estimation of the densities and the entropies of the attractors, the proposed B-spline method is suitable and this B-spline estimator can be used for online estimation in adaptive control of PDFs of the attractors as a future work.

\section{References}

Caudle, K. \& Wegman, E. [2009] "Nonparametric density estimation of streaming data using orthogonal series," Comput. Stat. Data Anal. 53, 3980-3986.

Cencov, N. [1962] "Estimation of an unknown density function from observations," Dokl. Akad. Nauk, SSSR, pp. 45-48.

Chua, L., Komuro, M. \& Matsumoto, T. [1986] "The double scroll family," IEEE Trans. Circ. Syst. $\mathbf{3 3}$, 1072-1118.

Chua, L., Yao, Y. \& Yang, Q. [1990] "Generating randomness from chaos and constructing chaos with desired randomness," Int. J. Circ. Th. Appl. 18, 215240.

Curry, H. \& Schoenberg, I. [1966] "On Pólya frequency functions IV: The fundamental spline functions and their limits," J. d'Analyse Mathématique 17, 71-107.

De Boor, C. [1972] "On calculating with B-splines * 1," J. Approx. Th. 6, 50-62.
Eckmann, J. \& Ruelle, D. [1985] "Ergodic theory of chaos and strange attractors," Rev. Mod. Phys. 57, 617.

Elbeyli, O., Hong, L. \& Sun, J. [2005] "On the feedback control of stochastic systems tracking prespecified probability density functions," Trans. Inst. Measur. Contr. 27, 319.

Er, G. [1998] "A method for multi-parameter PDF estimation of random variables," Struct. Safety 20, 25-36.

Er, G. [1999] "A consistent method for the solution to reduced FPK equation in statistical mechanics," Physica A 262, 118-128.

Er, G. [2000] "Exponential closure method for some randomly excited non-linear systems," Int. J. Nonlin. Mech. 35, 69-78.

Fraser, A. [1989] "Information and entropy in strange attractors," IEEE Trans. Inf. Th. 35, 245-262.

Günel, S. [2004] "An information theoretic approach to nonlinear control systems," PhD thesis, Dokuz Eylül University.

Günel, S. \& Savaci, F. [2005a] "Approximate stationary density of the nonlinear dynamical systems excited with white noise," IEEE Int. Symp. Circuits and Systems, 2005 (ISCAS 2005), pp. 4899-4902.

Günel, S. \& Savaci, F. [2005b] "Estimation of entropies of Chua's circuit," 13th Int. Workshop on Nonlinear Dynamics of Electronic Systems, Postdam, Germany.

Günel, S. \& Savaci, F. [2006] "Determination of the stationary state densities of the stochastic nonlinear dynamical systems," Int. J. Eng. Sci. 44, 14321447.

Guo, L. \& Wang, H. [2005] "PID controller design for output PDFs of stochastic systems using linear matrix inequalities," IEEE Trans. Syst. Man Cybern., Part B 35, 65-71.

Guo, L., Wang, H. \& Wang, A. [2008] "Optimal probability density function control for NARMAX stochastic systems," Automatica 44, 1904-1911.

Hong, W., Jin-Fang, Z. \& Hong, Y. [2005] "Multi-step predictive control of a PDF-shaping problem," Acta Automat. Sin. 31, 274-279.

Hwang, J., Lay, S. \& Lippman, A. [2002] "Nonparametric multivariate density estimation: A comparative study," IEEE Trans. Sig. Process. 42, 2795-2810.

Iyengar, R. \& Dash, P. [1978] "Study of the random vibration of nonlinear systems by the Gaussian closure technique," ASME Trans. Ser. E, J. Appl. Mech. 45, 393-399.

Kehtarnavaz, N. \& Nakamura, E. [1998] "Generalization of the EM algorithm for mixture density estimation," Patt. Recogn. Lett. 19, 133-140.

Lasota, A. \& Mackey, M. [1994] Chaos, Fractals, and Noise: Stochastic Aspects of Dynamics (Springer). 
Lázaro, M., Santamaría, I., Erdogmus, D., Hild, K., Pantaleón, C. \& Principe, J. [2005] "Stochastic blind equalization based on PDF fitting using Parzen estimator," Sign. Process., IEEE Trans. 53, 696-704.

Nakayama, K., Hirano, A. \& Sakai, T. [2003] "An adaptive nonlinear function controlled by estimated output PDF for blind source separation," Proc. ICA2003, Nara, Japan, pp. 427-432.

Pesin, Y. [1977] "Characteristic Lyapunov exponents and smooth ergodic theory," Russ. Math. Surv. 32, 55-114.

Pincus, S. [1991] "Approximate entropy as a measure of system complexity," Proc. Natl. Acad. Sci. USA 88, 2297.

Schumaker, L. [2007] Spline Functions: Basic Theory (Cambridge University Press).

Scott, D. [1992] Multivariate Density Estimation: Theory, Practice, and Visualization (Wiley-Interscience).

Shanmugan, K. \& Breipohl, A. [1988] Random Signals: Detection, Estimation, and Data Analysis (John Wiley \& Sons Inc.).

Sun, J. [2006] Stochastic Dynamics and Control (Elsevier Science Ltd.).
Suykens, J., Huang, A. \& Chua, L. [1997] "A family of $n$-scroll attractors from a generalized Chua's circuit," Archiv fur Elektronik und Ubertragungstechnik 51, 131-137.

Unser, M., Aldroubi, A. \& Eden, M. [1993] "B-spline signal processing. Part I: Theory," IEEE Trans. Sign. Process. 41, 821-833.

Wang, H. [2000] Bounded Dynamic Stochastic Systems: Modelling and Control (Springer Verlag).

Yalcin, M., Suykens, J. \& Vandewalle, J. [2004] "True random bit generation from a double-scroll attractor," IEEE Trans. Circ. Syst.-I: Regul. Pap. 51, 1395-1404.

Yalcin, M. [2007] "Increasing the entropy of a random number generator using $n$-scroll chaotic attractors," Int. J. Bifurcation and Chaos 17, 4471.

Yang, X. \& Li, Q. [2005] "On entropy of Chua's circuits," Int. J. Bifurcation and Chaos 15, 1823.

Zong, Z. \& Lam, K. [1998] "Estimation of complicated distributions using B-spline functions," Struct. Safety 20, 341-355.

Zong, Z. [2006] Information-Theoretic Methods for Estimating Complicated Probability Distributions (Elsevier Science Ltd.). 
Copyright of International Journal of Bifurcation \& Chaos in Applied Sciences \& Engineering is the property of World Scientific Publishing Company and its content may not be copied or emailed to multiple sites or posted to a listserv without the copyright holder's express written permission. However, users may print, download, or email articles for individual use. 